\title{
Security System with Three Way Authentication
}

\author{
Dharanendiran $\mathrm{C}^{1 *}$, Meenakshi $\mathrm{V}^{2}$ and Mohan $\mathrm{E}^{3}$
}

${ }^{1}$ Department of CSE Misrimal Navajee Munoth Jain Engineering College, Chennai, Tamil Nadu, India ${ }^{2}$ Department of ECE Misrimal Navajee Munoth Jain Engineering College, Chennai, Tamil Nadu, India

${ }^{3}$ P.T.Lee Chengalvaraya Naicker College of Engineering and Technology, Kanchipuram, Tamil Nadu, India

\begin{abstract}
Security is the prime concern of organizations and individuals these days. It could be a house or an office which needs to be modernized with latest security systems that are more secure and robust systematically. Conventional Lock with key methods are more vulnerable to replication of keys and physically getting broken, in order to overcome this, modern techniques like RFID and Mobile applications are used. Though the prior mentioned security systems are often implemented with only the alarms and cameras which may intimate the user about the breach and collect the evidences respectively, there aren't any counter-measures proposed to expel the thief out of the place. Therefore, the main objective of this paper is to deploy counter-measures to expel the thieves out of the place even after successful breach through the door/window along with the inherent functions of the already existing systems. This paper also proposes an effective handling way of a worst-case scenario, which prevents the deployment of countermeasures when a genuine user breaks through the door in emergency situations.
\end{abstract}

Keywords: Arduino; GSM module; Tranquilizers; Human odor sensor; RFID; Bluetooth (HC-05)

\section{Introduction}

New technologies and approaches are introduced into security systems with the aim of improving efficiency and minimizing errors in identifying the genuine users. In recent years there has been a tremendous growth in the smart homes and security automations. Efforts are being made to support and assist this growth with the usage of wireless technology. In most of the security systems, biometrics based authentication system exists, which lacks flexibility and are more prone to hacks now.

\section{Scope and need for system}

A lot of consistent changes have been introduced in home and industrial security automation domain in the past decade. One of the significant steps is the usage of Iris and fingerprint scanning techniques. But as time goes by, these techniques are compromised by rogue hackers and eventually by federal agencies. In order to curb these activities, we have introduced a new method of authentication with good flexibility to the user by using multiple ways of authentications which are RFID based, Bluetooth based and Human odor based.

- RFID: Radio Frequency Identification uses electromagnetic chips to read and write the tags attached to objects like keychains. In this proposed system a 16-bit key is stored in the tag for verification to operate door lock.

- Bluetooth: This is a short-ranged wireless technology which is used to transfer data among fixed and mobile devices. In this proposed system an Android application has been developed with cloud authentication with which the door lock can be operated via Bluetooth.

- Human odor: Every human being will have a unique odor $[1,2]$. With this odor we have implemented an experimental authentication technique as a worst case scenario in this proposed system.

- Counter-measures: In this proposed system a non-fatal dose of tear gas or tranquilizers are used to expel the intruders out or to capture them. Authentication via Human odor prevents this countermeasure to act upon genuine users.

Mammals such as mice and humans are known to have unique, genetically determined body odors, called odortypes, which act something like olfactory nametags, helping distinguish to individuals from one another [1].

Just as everyone has their own individual and unique fingerprint, so does every individual when it comes to body odor [2].

"Security Systems for Smart Home based on GSM technology" in which they have used PIR sensor to monitor for intruders, Temperature and LPG sensors. The users are communicated via GSM modules and the proposed system is controlled by an Atmega644p microcontroller [3].

"Implementation of Home Security System using GSM module and Microcontroller" has proposed a security system using magnetic door sensor and GSM modem with LEDs [4].

"Wireless Home and Industrial Automation Security System Using GSM" has proposed a system with two modes. This system consist of smoke, temperature, GSM and PIR modules. In external mode all the modules are activated and any suspicious situations are notified to the user by SMS. In internal mode all the modules except the PIR are activated in order to curb unwanted detections on genuine users [5].

"Automated Advanced Industrial and Home Security Using GSM and FPGA" has proposed to control various home appliances and to detect security breaches and fire detection through SMS. This system

*Corresponding author: Dharanendiran Chandrababu, Department of CSE Misrimal Navajee Munoth Jain Engineering College, Chennai, Tamil Nadu, India, Tel: +9124962270; E-mail: cdharanbabu@gmail.com

Received September 26, 2017; Accepted October 11, 2017; Published October 16, 2017

Citation: Dharanendiran C, Meenakshi V, Mohan E (2017) Security System with Three Way Authentication. Adv Robot Autom 6: 176. doi: 10.4172/21689695.1000176

Copyright: () 2017 Dharanendiran C, et al. This is an open-access article distributed under the terms of the Creative Commons Attribution License, which permits unrestricted use, distribution, and reproduction in any medium, provided the original author and source are credited. 
is also capable of informing Fire/Police departments about the incident [6].

"Microcontroller Based Home Security System with GSM Technology". This proposes a keypad based lock and an android interface to control the microcontroller system via Bluetooth. This system incorporates GSM technique to inform the users about intruder activity and fire break outs [7].

\section{Disadvantages}

"Detection and Classification of Human Body Odor Using an Electronic Nose" proposes each human being has a unique odor [8].

\section{Existing System}

The proposed system is controlled by an Atmega644p microcontroller. It collects information from the sensors, makes a decision and sends SMS to a corresponding number by using a GSM modem. If it finds any interruption in its sensors (for example IR sensor) then microcontroller will send a SMS to the home owner. In the same way if the temperature is increased above certain point or gas sensor sensors is ON, a SMS will be sent to the home owner 'Fire at home' giving the indication of fire. The outputs of all the sensors are connected to ADC. One IR is connected at window and other is at door. The entry from the window is treated as unauthorized entry and entry from door is treated as authorized entry [3].

The security system uses the Yawcam (Yet another WebCAM Software) software to interface the web camera. Yawcam is Multilingual Java software for viewing and transferring images or videos from a webcam. Yawcam is a versatile tool for web camera utilization. With yawcam it is possible to observe the web camera, take snapshots or stream the video to the internet. Snapshots or video can be uploaded to the web using your own PC as a server [3].

The system is fully controlled by the microcontroller and the microcontrollers will continuously monitor the sensors, detector and GSM modem [5].

This system construct with 8051 microcontroller which contains a piece of code for a specific action. The action will be taken by the GSM modem i.e., to alert an owner about intruder or danger to house. That device should be installed on the door, as the intruder opens the door the magnet which is situated in the door go away from Relay so LED glows with RED light and the appropriate action will be taken by GSM modem [5]. The working of this system is illustrated in Figure 1.

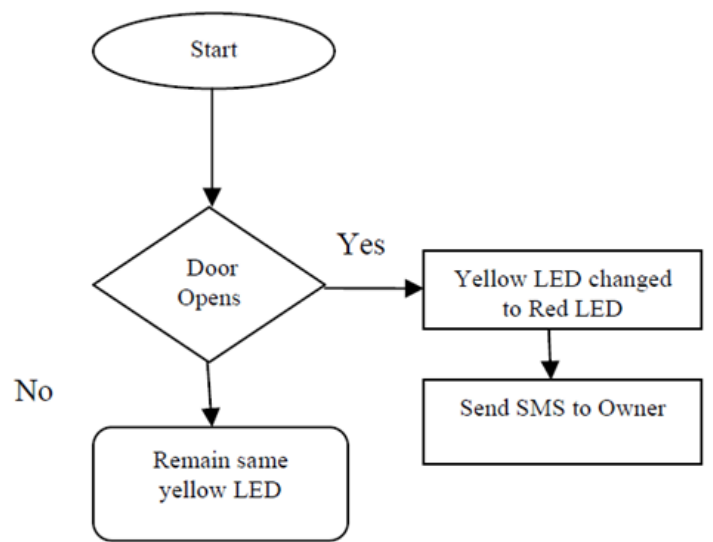

Figure 1: Flow chart.
- Most of the existing systems alone uses PIR sensor for motion tracking which is often inaccurate and reduces the reliability of the system.

- The camera used in existing systems is neither infrared or night vision supported and are static in nature.

- The static cameras used are not much of use for live telecasting and snapshotting purposes, since motion tracking cameras are required to monitor the movement and to capture them.

- A simple SMS sent to the user's phone may not attract their attention.

- Buzzers installed inside the house are of no use when there is no one inside the house or the office premises.

- There are no contingency mechanisms implemented in any of the existing systems like "what if the user loses the key and if he wants to enter the home by breaking the door?" the system without a contingency mechanism will identify the user as an intruder.

\section{Proposed System}

The proposed system consists of three types of authentications which are using RFID tags to operate the door lock, using android application based on cloud authentication to operate the door lock and using odor to detect the user as a contingency authentication method. Intruders trying to enter the place are expelled/captured using tear gas or tranquilizers, respectively. The proposed system can detect LPG leakage. This system incorporates a night vision camera with tilting and rotating facilities which may be used for recording videos based on the motion and also for capturing images.

The RFID tags/keys are of two types in which one is a master key and the other is a guest card. The user/administrator is informed with a SMS whenever a guest card is authenticated.

If a guest wants to enter the place without a tag, then he/she may use the android application. First the guests need to register themselves to the cloud server. On successful approval of the user/administrator the guest could login into the android application and get access to the place. This authentication method depends on the Bluetooth technology.

The administrator is informed via SMS after successful logging in of the guest.

Hazardous situations like detection of intruders or LPG leakage are informed to the user by means of a buzzer through the android application named TWASS in their phone. The application also facilitates the user to SMS the police/fire department by selecting the appropriate location of their home during installation of the application.

\section{Advantages}

- The proposed system is flexible with the user as it has more than one way of authentication.

- Since it uses both magnetic door and PIR sensors, the accuracy is highly improved.

- Provided cloud authentication with the Bluetooth authentication method adds an additional layer of security.

- The usage of HD night vision camera with motion tracking 
capabilities improves the viability and reliability of the live monitoring facility.

- Interactive Android applications are used to interpret the codes sent to the mobile phone from the system, so that the application may turn on the appropriate alerts to grab the user's attention by buzzing through the phone.

- Using phone as a buzzer allows the user to get attention of hazardous situations in his/her premises from anywhere.

- This system consists of counter measures and contingency authentication method implemented to defy the counter measures on genuine users in emergency situations.

\section{System Architecture}

The basic block diagram of the system architecture is as shown in Figure 2 shown below. This diagram displays the elements of the project involving sensors and microcontroller.

\section{Android application}

Android is an open source operating which supports wide variety of programs to run on it. These programs are known as android applications.

\section{Short Messaging Service (SMS)}

This is a standard text messaging service which is used to exchange text messages from more than one mobile devices.

\section{Microcontroller}

A microcontroller is a processor which is specifically developed for a particular purpose unlike a microprocessor which operates in a generic way.

\section{Bluetooth and GSM}

Bluetooth is a short ranged wireless technology which is used to transfer the data between mobile and fixed devices. Whereas GSM (Global system for mobile networks) is a second generation cellular network.

\section{Hardware Requirements}

This section describes the sensors, microcontroller and GSM module used in the implementation of hardware.

\section{Arduino UNO R3}

Arduino UNO R3 is the microcontroller proposed to be used in this system, because of its cheaper price, flexibility and reliability. This controls all the sensors and other components of the system (Figure 3).

\section{Magnetic door sensors}

Magnetic door sensors also known as reed switches. It is an electrical switch which is operated by the magnetic field. The switch is actuated by bringing a magnet near to the switch. When the magnet is pulled away the switch will go back to its original position (Figure 4).

\section{RFID}

Radio-frequency identification (RFID) uses electromagnetic fields to automatically identify and track tags attached to objects. The tags contain electronically stored information. There are two types of RFID tags which are Administrator tag and Guest tag (Figure 5).

\section{PIR sensor}

An infrared sensor is an electronic instrument which is used to measure the heat being emitted by an object and detecting motion. IR sensors are used to detect the entry of the intruder. The working of the PIR sensor is illustrated in Figure 6.

\section{Servo motors}

Servos are controlled by sending an electrical pulse of variable width, or pulse width modulation (PWM), through the control wire.

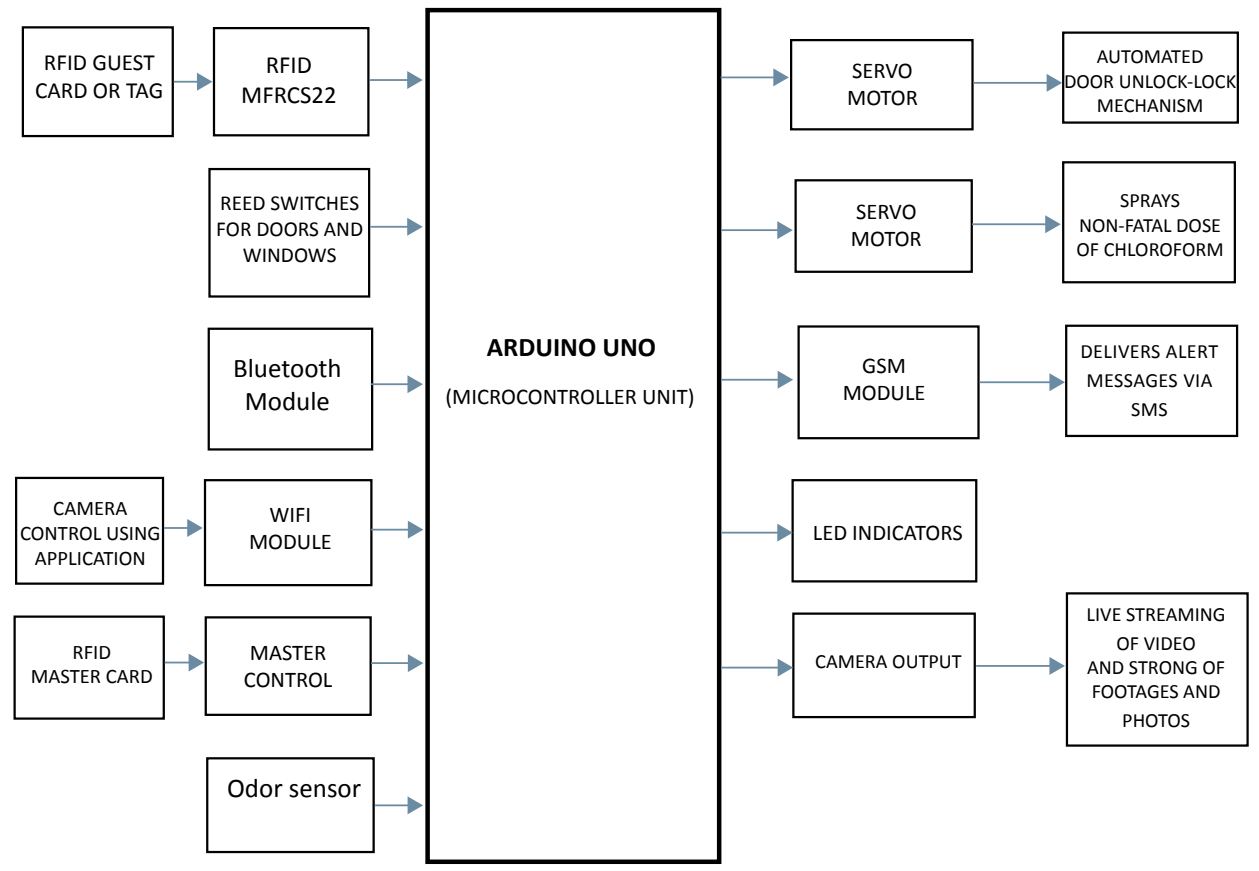

Figure 2: Block diagram of the proposed system. 


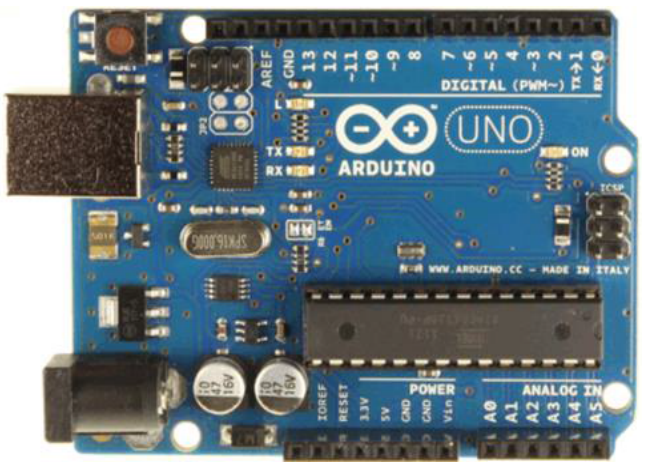

Figure 3: Arduino UNO R3 micro-controller.

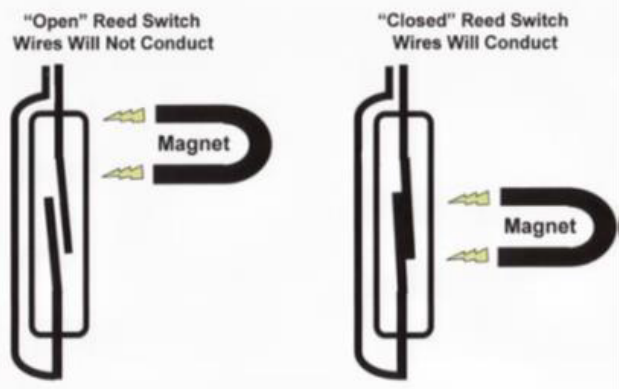

Figure 4: Reed switches.

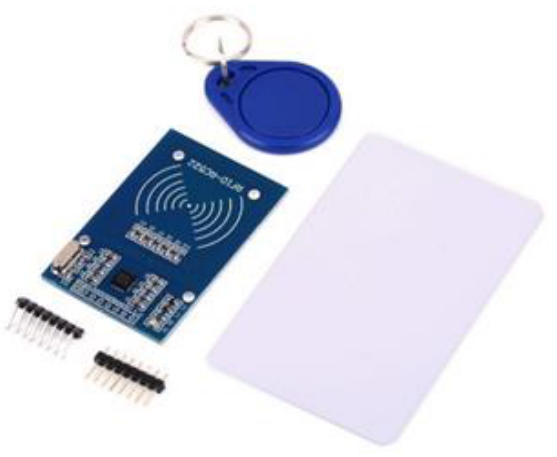

Figure 5: RFID RC522 with tags.

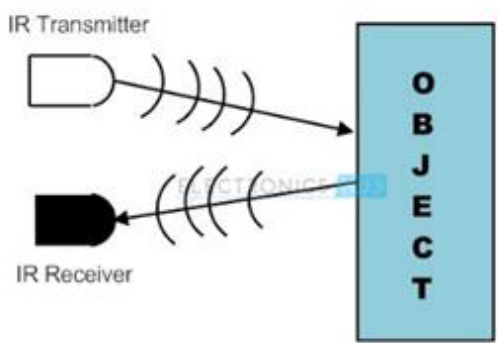

Figure 6: IR sensor architecture.

These motors are capable of rotating up to 180 degrees. In the proposed system the servos are used to drive automatic door locks and tear gas or tranquilizer cans.

\section{GSM and Bluetooth modules}

Bluetooth module is used for authentication and GSM module is used for communication purpose with the user. Here the Bluetooth and GSM modules used are HC-05 and SIM900A respectively. Figure 7 illustrates the SIM900A GSM module.

\section{IP camera}

IP camera facilitated with HD video recording, live streaming and snapshot facilities used in this system. This camera has the facility to get rotated and tilted wirelessly through the android application.

\section{MQ2/LPG sensor}

This MQ2 sensor is a gas sensor which is used to detect smoke and LPG leakage detection in the proposed system.

\section{Human odor sensor}

The E-nose [8] also known as the electronics nose is used to detect the unique odor from each human. This sensor produces a binary value which can be used for verification of the genuine user.

\section{Software Requirements \\ TWASS}

Abbreviated as Three Way Authentication Security System this android application facilitates the guest to enter the place without RFID tags. The guest has to first register with the administrator who is provided with a unique administrator ID. In this case the owner of the place is the administrator. After the approval from the administrator the guest could login to the application to gain access to the place.

\section{TWASS Admin}

This application is used to notify the user when intruders or LPG leakage or fire is detected. It alerts the user by buzzing and vibrating the mobile phone. It also allows the user to send SMS to the nearby police/ fire stations.

\section{Flow Chart}

The following flowchart as shown in Figure 8a-d displays the detailed step by step process taking place in the proposed project.

Step 1: The LPG/smoke sensor checks for leakage of LPG and smoke, if there are any then it jumps to step 4, else the control moves to step 2.

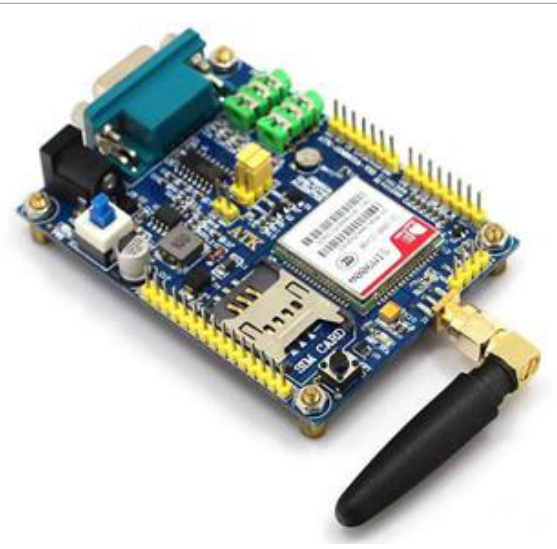

Figure 7: SIM900A GSM Module. 
Step 2: The magnetic door sensor remains in LOW state till the door is closed. When the door is opened it returns HIGH to the Arduino and moves to step 3.

Step 3: The Arduino checks for TRUE if in anyone of the three authentication methods which are FALSE typically, if none of them are true it will move to step 4, else if any of the methods returns TRUE move to step 5 , the three authentication methods are.

\section{RFID authentication}

When the master tag is used, the door will be opened after successful verification.

When the guest tag is used, the door will be opened after successful verification and a SMS is sent to the user.

\section{Bluetooth authentication}

The guest registers themselves using the administrator ID using the TWASS application.

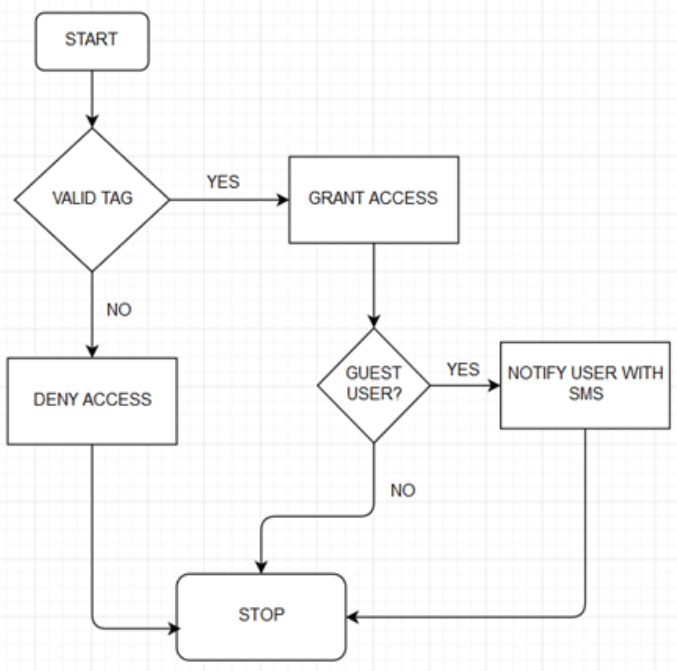

Figure 8a: Authentication with RFID

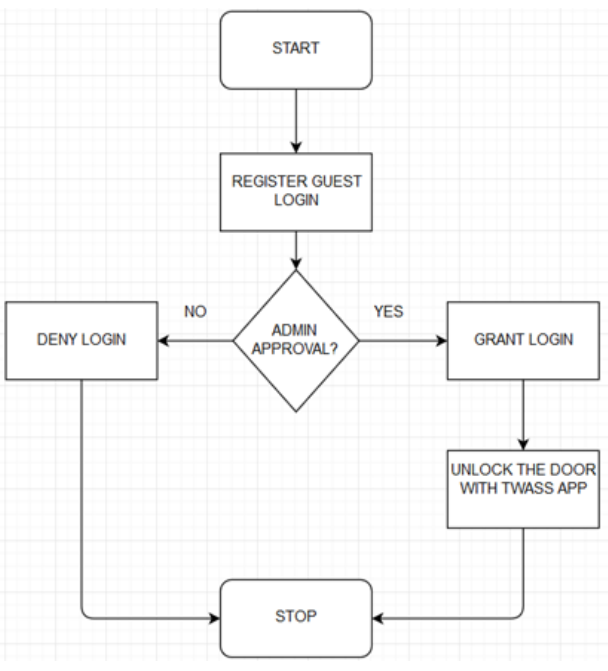

Figure 8b: Authentication with Bluetooth.
On the approval the administrator (i.e., the user), the guest can login and get access to the place.

\section{Human odor authentication}

If it happens for a genuine user to break the door and enter the house in emergency situations the body odor of the user is verified for the authentication, if the verification succeeds counter-measures may not be initiated.

Step 4: Send an appropriate emergency message to the user regarding the situation.

Step 5: Wait until the door closes and move to step 1.

\section{Result Analysis}

Thus this system provides more secure and sophisticated way of authentications with GSM connectivity and interactive android application. The flexibility of the system is extended to a higher degree by implementing three types of authentications. This system provides a unique and effective way of handling critical situations like identifying intruders and genuine users followed by deployment of counter-

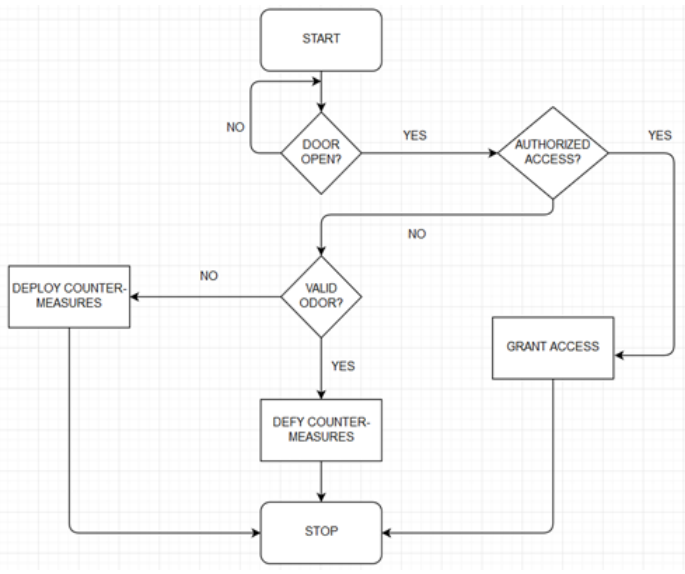

Figure 8c: Authentication with Human odor

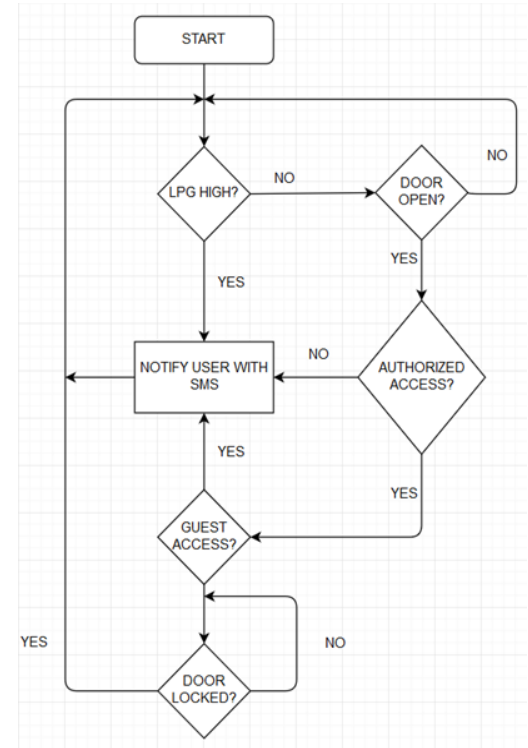

Figure 8d: Flow-Chart 
measures in the case of intruders. The android application developed alerts the user in an effective way unlike conventional SMSs which are laconic and sometimes goes unnoticed. Apart from these features, inherent functions of the earlier systems like LPG/Smoke detection are also included in this system. Thus the proposed system is robust, cheaper, reliable and flexible for implementation.

\section{Future Enhancements}

The mobile application developed for android can be developed for other platforms like iOS, Windows etc. RFID chips can be embedded into the conventional lock and keys so that they may detect usage of duplicate keys and they may provide more robust locks. Remote access to the door locks can be implemented using advanced microcontrollers in the future.

\section{References}

1. Thompson A (2008) Your Odor: Unique as Fingerprint. Live Science.

2. Lees K (2013) Every Person has their Own Unique Scent. Science World Report.
3. Bangali J, Shaligram A (2013) Design and Implementation of Security Systems for Smart Home based on GSM technology. International Journal of Smart Home 7: 201-208.

4. Parab AS (2015) Implementation of Home Security System using GSM module and Microcontroller. International Journal of Computer Science and Information Technologies 6: 2950-2953.

5. Anandan R, Karthik B, Kumar KTVU (2015) Wireless Home and Industrial Automation Security System Using GSM. Journal of Global Research in Computer Science 4: 55-59.

6. Chintaiah N, Rajasekhar K, Dhanraj V (2011) Automated Advanced Industrial and Home Security Using GSM and FPGA. International Journal of Computer Science and Information Technologies 2: 1598-1602.

7. Hasan R, Khan MM, Ashek A, Rumpa IJ (2015) Microcontroller Based Home Security System with GSM Technology. Open Journal of Safety Science and Technology 5: 55-62.

8. Wongchoosuk C , Lutz M, Kerdcharoen T (2009) Detection and Classification of Human Body Odor Using an Electronic Nose. Sensors - Open Access Journal 9: 7234-7249. 